\title{
Exploring the Potential of Realtime Haptic Feedback during Social Interactions
}

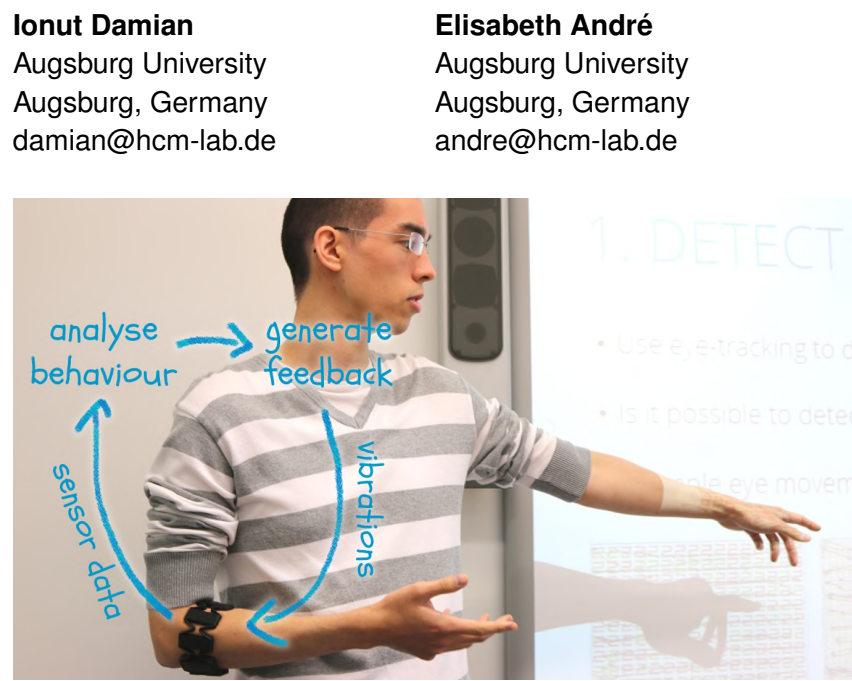

Figure 1: System prototype meant to support user in monitoring and improving nonverbal behaviour using haptic feedback delivered by a Myo armband during a public speech.

This is the author's version of the work. It is posted here for your personal use. Not TEl '16. Februn. The definitive Version of Record was putys

TEI 16, February 14-17, 2016, Ehrohoven, Netherlands

rights licensed to ACM

http//dx doi.org/10.1145/2839462.2856519

\begin{abstract}
We explore the use of haptic feedback to deliver supportive information during social interactions in realtime. In an exploratory user study, we investigated perceptual limitations of vibration patterns during a conversation between peers. The results from this study have then been used to develop a system for providing users with realtime information regarding the quality of their nonverbal behaviour while engaged in a public speech.
\end{abstract}

\section{Author Keywords}

haptic feedback; realtime; social interaction; nonverbal behaviour; signal processing

\section{ACM Classification Keywords}

H.5.m. [Information Interfaces and Presentation (e.g. $\mathrm{HCl}$ )]: Miscellaneous

\section{Introduction}

The ability of man to attend to secondary stimuli whilst being engaged in a primary task has been heavily studied in the past. The vast majority of models of attention which have been proposed in the last century have one thing in common, it is easier for man to switch or attend to stimuli which do not compete for the same cognitive structures with the primary task. For example, we have no problem listening to music while cooking but we usually turn the music 
down when engaging in a conversation with another person. These structural interferences [8] mean that it is easier for us to handle tasks if they involve different modalities [2]. In the example above, it is easier to cook (visual) and listen to music (auditory) than to converse (auditory) and listen to music (also auditory). Following this line of thought, it is reasonable to state that, in a social interaction which stresses mostly our auditory and visual processing paths, haptic feedback would be a fitting secondary task.

Other researchers already successfully made use of haptic feedback to help users learn to play the violin [16], convey nonverbal communication cues to the visually impaired [10] help pedestrians navigate [12] and provide instructions during physical activities [13]. When it comes to feedback in social situations, most work concentrates on visual or auditory feedback $[3,11,15]$, making haptic feedback a still relatively unexplored topic.

In this work, we propose to use haptic feedback as an information delivery mechanism during social interactions, e.g. public speaking. To this end we conducted an exploratory user study to find out how well users can perceive haptic feedback while engaged in a social interaction. Based on the findings from the study, we developed a system prototype (Figure 1) capable of providing realtime haptic feedback to inform the user of her or his nonverbal behaviour whilst delivering a public speech.

\section{Background}

Haptic feedback involves the delivery of messages through systematic arousal of the user's skin. The most common approach found in human-computer interaction is vibration feedback. For example, our phones vibrate when we get a call or our game controller vibrates when we perform an action inside the game environment.
Outside of technology-enhanced systems, the sense of touch plays a crucial role in our day-to-day lives. It enables accurate and rapid manipulation as well as interaction with physical objects. Furthermore, it allows us to quickly gather information from physical objects, such as solidity, texture and temperature while also playing an important role in social interactions where it communicates emotions [4].

The main advantage of haptic feedback is that social interactions primarily only involve the visual and auditory channel. This makes haptic feedback an ideal candidate to accompany such primary tasks as it is less prone to structural interferences and thus, less likely to disrupt the primary task. However, the disadvantage of haptic feedback is its reduced bandwidth. The spatial resolution of our sense of touch is mostly limited by the distribution of receptive fields on the skin. The finger, which is considered one of the most receptive part of the body [17], has receptive fields measuring upwards of $2 \mathrm{~mm}$ in diameter [6]. Regarding the temporal resolution, studies show that humans are able to differentiate between haptic stimuli 2 - 40 ms apart, depending on location (Kenshalo 1978 in [9]). However, these values are upper bounds which are very hard to reach due to limited attention and processing resources [1, 5, 9]. More concrete thresholds are provided by Tan et al. [14], who found that humans can optimally perceive 2-3 items per second using the haptic channel whereas Kaaresoja and Linjama [7] suggest a $50 \mathrm{~ms}$ limen for reliable perception of vibrations.

\section{Exploratory User Study}

To inform the design of our system prototype for behaviour monitoring and regulation using haptic feedback, we conducted a small scale user study. 10 participants ( 8 male and 2 female) aged between 21 and 33 (mean 27.4) tried out a simple haptic feedback system. The aim of the study 


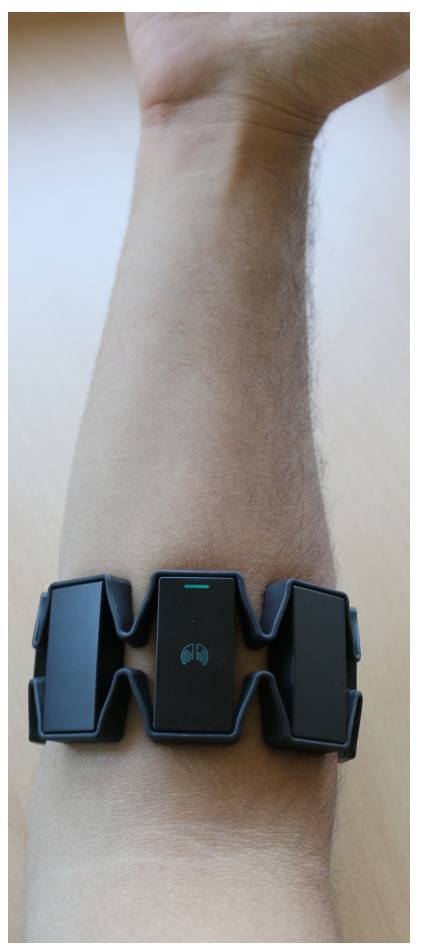

Figure 2: Position of Myo armband on user's forearm was to get a better image of our body's haptic perception in a social scenario by measuring how well users are able to detect different types of haptic feedback while engaged in a conversation.

\section{Apparatus}

The apparatus consisted of a Myo armband ${ }^{1}$ strapped to the user's forearm. While the Myo's main selling point is the EMG sensor, for the purpose of this study we only used its vibration-actuator. Using two parameters, intensity (value ranging from 1 to 250) and duration (in milliseconds), the Myo can be programmed to deliver various types of vibrations. To maximize the haptic sensation, the Myo was positioned with the vibration module (the one with the LED) on the underside of the forearm (see Figure 2). For the study, we implemented a total of 15 vibration patterns as detailed in Table 1. The starting values of the vibration scales have been chosen following various pre-tests which showed that the Myo (running firmware 1.2.955) can not produce vibrations shorter than $10 \mathrm{~ms}$ and of an intensity smaller than 60.

\section{Procedure}

After receiving a short demonstration of the first five vibration patterns (P1 - P5), the experimenter engaged the participants in a casual conversation for approximately 3 minutes. During this time, the Myo would vibrate 6 times, once every 30 seconds using a pattern chosen at random [18] from the first five patterns in Table 1 (P1 - P5). The participants were instructed to mark the vibration occurrence and pattern in a questionnaire as soon as they felt it and then continue with the conversation.

In the second part of the study, after the conversation had finished, the experimenter played back the two vibration

\footnotetext{
${ }^{1}$ https://www.thalmic.com/myo/
}

\begin{tabular}{|c|c|c|c|}
\hline ID & Duration & Intensity & Description \\
\hline P1 & 500 & 150 & $\begin{array}{l}\text { Single short vibra- } \\
\text { tion }\end{array}$ \\
\hline P2 & $500,500,500$ & $150,0,150$ & $\begin{array}{l}\text { Double vibration } \\
\text { with } 500 \text { ms break }\end{array}$ \\
\hline P3 & 1000 & 150 & $\begin{array}{l}\text { Single long vibra- } \\
\text { tion }\end{array}$ \\
\hline P4 & 500 & 250 & $\begin{array}{l}\text { Single strong short } \\
\text { vibration }\end{array}$ \\
\hline P5 & $500,500,500$ & $100,250,100$ & "wave" pattern \\
\hline SD & $\begin{array}{l}10,20,30 \\
50,100\end{array}$ & 150 & $\begin{array}{l}\text { Vibration duration } \\
\text { scale }\end{array}$ \\
\hline $\mathrm{SI}$ & 500 & $\begin{array}{l}60,70,80 \\
90,100\end{array}$ & $\begin{array}{l}\text { Vibration intensity } \\
\text { scale }\end{array}$ \\
\hline
\end{tabular}

Table 1: Vibration patterns. Duration is in milliseconds and intensity is a value from 1 to 250 .

scales (SD and SI). After each scale, the participants were asked how many vibrations they felt. During this part of the experiment, participants wore noise-cancelling headphones as the Myo's vibration-actuator also produces a noticeable sound when turned on which, at times, is more perceivable than the vibration itself.

Results

Upon analysis of the results, we noticed that the participants were able to recognize the patterns $81.6 \%$ of the time. The confusion matrix in Figure 3 shows that they had a hard time recognizing vibration intensity, often times confusing P4 with P1. Furthermore, despite the relatively high recognition rate, during the study most of the participants struggled to identify the vibration pattern stating that "this is harder than I thought".

Observations during the study revealed that the feedback 


\begin{tabular}{|c|c|c|c|c|c|}
\hline & P1 & P2 & P3 & P4 & P5 \\
\hline P1 & 12 & & & & \\
\hline P2 & & 12 & & & \\
\hline P3 & & 1 & 10 & & \\
\hline P4 & 5 & & 1 & 6 & \\
\hline P5 & 1 & 2 & & & 9 \\
\hline
\end{tabular}

Figure 3: Confusion matrix showing recognitions of haptic feedback events. Each event occurred 12 times during the study.

did not disturb the participants' speech, with some even missing a few vibrations. This effect is confirmed by the participants themselves. When asked whether the haptic feedback disturbed the conversation, most users said no. P3 stated "It may actually have been too subtle as I was very involved in the conversation." P10 was more analytical, comparing it to visual feedback: "Since the vibrations use a different channel ... I was using my eyes to look at you ... I didn't find [the feedback] disturbing."

Regarding the vibration scales, the participants felt on average the last 2.2 vibrations from the duration scale, and the last 4 from the intensity scale. This translates to an approximated perception limen for the forearm of just under $50 \mathrm{~ms}$ for duration and 70 for intensity. These findings are in line with the study by Kaaresoja and Linjama [7] suggesting that the position of the Myo does not have a strong impact on the threshold. However, both results differ from the 2-40 ms distal threshold mentioned by Kenshalo [9]. One possible explanation is that the minimum temporal distance between two stimuli does not correlate strongly with the minimum duration of a stimulus. The difference might also be caused by the fact that the vibration-actuators in both the Myo and the mobile phone used in [7] are not in direct contact with the user's skin.
Overall, the study revealed that haptic feedback is promising as it does not seem to disturb the social conversation. However, recognition problems with the more complex patterns suggest that feedback needs to be kept simple.

\section{Prototype}

Based on the data gathered from the exploratory study, we developed a system prototype capable of delivering haptic feedback during a public speech to inform the user of the quality of her or his gestural behaviour and help improve it. In this regard, the system borrows from the augmented social interaction concept presented by Damian et al. [3]. In their work, they combine social signal processing techniques with personal augmentation methods to project social behaviour aids in the user's field of view using a Google Glass. The aim of their work is to increase the user's awareness of their own nonverbal behaviour.

In our work, we use a Myo armband to analyse the user's nonverbal behaviour, more specifically the arm movements, in realtime. We trigger a vibration in the Myo if the user either moves too much or not enough. The aim here is to assist the user in delivering a compelling and enticing speech. The rest of this section will provide more details on both behaviour analysis and feedback delivery.

\section{Behaviour Analysis}

The behaviour of the user's arm is processed in real time using a signal processing pipeline. Acceleration and magnetometer data is extracted from the Myo to compute the dynamic acceleration ${ }^{2}$ of the user's arm 10 times per second. Using the dynamic acceleration, we then compute the velocity and the arm displacement over a frame window

${ }^{2}$ The dynamic acceleration is computed from the acceleration by compensating for the gravity vector: http://www.varesano.net/blog/fabio/ simple-gravity-compensation-9-dom-imus 


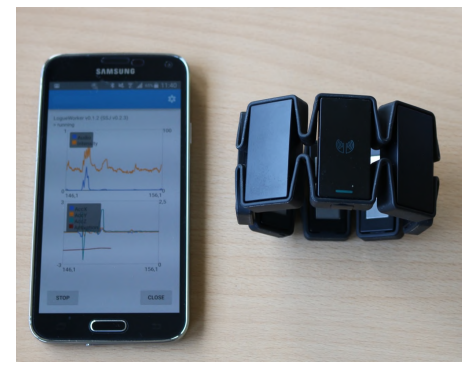

Figure 4: System prototype running on an android smartphone of 5 seconds. Finally, the resulting value is filtered using a moving average with a window length of 50 seconds to eliminate any sudden movements or noise caused by either environmental factors or the vibration feedback itself. The resulting value (referred to as arm activity) measures how much the user's arm moved over a 5 second window.

\section{Feedback}

The arm activity is classified using an empirically refined threshold approach. If the value is either too low or too high, a vibration on the Myo is triggered. More specifically, if the activity is lower than 0.8 , a single low intensity $500 \mathrm{~ms}$ long vibration is generated. On the other hand, if the activity is higher than 2.0, two 500 ms low intensity vibrations 500 ms apart are triggered. We chose the single and double vibration patterns as these yielded the best recognition rate in our exploratory study. Initially, the feedback intensity is set at 70 , which is slightly above the reported perception limen so that short dips outside the activity thresholds are less disrupting. However, if the user does not react to the feedback, cognitive overload might inhibit the perception of subtle feedback (due to limited spare capacity [8]) and more powerful stimuli may be needed to capture attention. To this end, every 10 seconds after the initial feedback has been delivered, the vibration is repeated and the intensity is gradually increased until the user adjusts.

The prototype is working on an android smartphone which is connected to a Myo armband (see Figure 4) and initial informal tests are promising. However, a more in-depth evaluation is needed to make a clear statement regarding the effectiveness and disruptiveness of the feedback. A live demo of the system prototype is planned to be shown at TEI. Furthermore, the software is open source and freely available for download ${ }^{3}$.

\footnotetext{
${ }^{3}$ http://hcm-lab.de/logue
}

\section{Conclusion}

We report on our efforts in investigating the feasibility of haptic feedback to deliver supportive information during social interactions. To this end, we explored the limits of human perception for recognizing haptic patterns while engaged in a conversation. We found that users are able to perceive haptic feedback whilst engaged in a social interaction and that the feedback does not seem to disturb the user or the interaction. However, users did have difficulty differentiating between complex vibration patterns suggesting that haptic feedback needs to be kept simple. We also describe our initial prototype capable of informing the user of her or his own nonverbal behaviour in realtime through the use of haptic feedback. Despite the perceptual limitations revealed by our study, we propose a feedback mechanism which generates awareness of one's own arm movement quality using just two simple vibration patterns.

As part of our future work, we aim to thoroughly investigate the effects (both positive and negative) such a system can have on the user's behaviour, the interaction itself and other interlocutors.

\section{Acknowledgements}

The work was partially funded by the German Federal Ministry of Education and Research (BMBF) under the project "GLASSISTANT".

\section{REFERENCES}

1. James C. Bliss, Hewitt D. Crane, Phyllis K. Mansfield, and James T. Townsend. 1966. Information available In brief tactile presentations. Perception \& Psychophysics 1, 4 (1966), 273-283.

2. Lee R. Brooks. 1968. Spatial and verbal components of the act of recall. Canadian Journal of Psychology/Revue canadienne de psychologie 22, 5 
(1968), 349-368.

3. Ionut Damian, Chiew Seng Tan, Tobias Baur, Johannes Schöning, Kris Luyten, and Elisabeth André. 2015. Augmenting Social Interactions: Realtime Behavioural Feedback using Social Signal Processing Techniques. In Proceedings of the 33rd Annual ACM Conference on Human Factors in Computing Systems (CHI). 565-574.

4. Matthew J. Hertenstein, Dacher Keltner, Betsy App, Brittany A. Bulleit, and Ariane R. Jaskolka. 2006. Touch communicates distinct emotions. Emotion (Washington, D.C.) 6 , 3 (2006), 528-533.

5. John W. Hill and James C. Bliss. 1968. Perception of sequentially presented tactile point stimuli. Perception \& Psychophysics 4, 5 (1968), 289-295.

6. Roland S. Johansson. 1978. Tactile sensibility in the human hand: Receptive field characteristics of mechanoreceptive units in the glabrous skin area. The Journal of Physiology 281, 1 (1978), 101-125.

7. Topi Kaaresoja and Jukka Linjama. 2005. Perception of Short Tactile Pulses Generated by a Vibration Motor in a Mobile Phone. In First Joint Eurohaptics Conference and Symposium on Haptic Interfaces for Virtual Environment and Teleoperator Systems. 471-472.

8. Daniel Kahneman. 1973. Attention and effort. Prentice Hall, Englewood Cliffs.

9. Jack M. Loomis. 1981. Tactile pattern perception. Perception 10, 1 (1981), 5-27.

10. Troy McDaniel, Sreekar Krishna, Vineeth Balasubramanian, Dirk Colbry, and Sethuraman Panchanathan. 2008. Using a haptic belt to convey non-verbal communication cues during social interactions to individuals who are blind. In 2008 IEEE
International Workshop on Haptic Audio visual Environments and Games (HAVE 2008). 13-18.

11. Eyal Ofek, Shamsi T. Iqbal, and Karin Strauss. 2013. Reducing Disruption from Subtle Information Delivery During a Conversation: Mode and Bandwidth Investigation. In Proceedings of the SIGCHI Conference on Human Factors in Computing Systems. ACM, 3111-3120.

12. Martin Pielot, Benjamin Poppinga, Wilko Heuten, and Susanne Boll. 2011. A Tactile Compass for Eyes-Free Pedestrian Navigation. In Human-Computer Interaction - INTERACT 2011. Lecture Notes in Computer Science, Vol. 6947. Springer Berlin Heidelberg, Berlin, Heidelberg, 640-656.

13. Daniel Spelmezan, Mareike Jacobs, Anke Hilgers, and Jan Borchers. 2009. Tactile motion instructions for physical activities. In Proceedings of the SIGCHI Conference on Human Factors in Computing Systems. 2243-2252.

14. Hong Z. Tan, Nathaniel I. Durlach, Charlotte M. Reed, and William M. Rabinowitz. 1999. Information transmission with a multifinger tactual display. Perception \& Psychophysics 61, 6 (1999), 993-1008.

15. M. Iftekhar Tanveer, Emy Lin, and Mohammed Hoque. 2015. Rhema: A Real-Time In-Situ Intelligent Interface to Help People with Public Speaking. In Proceedings of the 20th International Conference on Intelligent User Interfaces. 286-295.

16. Janet van der Linden, Erwin Schoonderwaldt, Jon Bird, and Rose Johnson. 2011. MusicJacket-Combining Motion Capture and Vibrotactile Feedback to Teach Violin Bowing. IEEE Transactions on Instrumentation and Measurement 60, 1 (2011), 104-113. 
17. Sidney Weinstein. 1968. Intensive and Extensive Aspects of Tactile Sensitivity as a Function of Body

Part, Sex and Laterality. In International Symp On Skin Senses.

18. E. J. Williams. 1949. Experimental Designs Balanced for the Estimation of Residual Effects of Treatments. Australian Journal of Chemistry 2, 2 (1949), 149. 